\title{
Dimensional capability of selected 3DP technologies
}

\author{
Younss Ait Mou and Muammer Koc \\ Division of Sustainable Development, College of Science and Engineering, Hamad Bin Khalifa University, Qatar Foundation, \\ Education City, Doha, Qatar
}

\begin{abstract}
Purpose - This paper aims to report on the findings of an investigation to compare three different three-dimensional printing (3DP) or additive manufacturing technologies [i.e. fused deposition modeling (FDM), stereolithography (SLA) and material jetting (MJ)] and four different equipment (FDM, SLA, MJP 2600 and Object 260) in terms of their dimensional process capability (dimensional accuracy and surface roughness). It provides a comprehensive and comparative understanding about the level of attainable dimensional accuracy, repeatability and surface roughness of commonly used 3DP technologies. It is expected that these findings will help other researchers and industrialists in choosing the right technology and equipment for a given 3DP application.

Design/methodology/approach - A benchmark model of $5 \times 5 \mathrm{~cm}$ with several common and challenging features, such as around protrusion and hole, flat surface, micro-scale ribs and micro-scale long channels was designed and printed repeatedly using four different equipment of three different 3DP technologies. The dimensional accuracy of the printed models was measured using non-contact digital measurement methods. The surface roughness was evaluated using a digital profilometer. Finally, the surface quality and edge sharpness were evaluated under a reflected light ZEISS microscope with a $50 \times$ magnification objective.

Findings - The results show that FDM technology with the used equipment results in a rough surface and loose dimensional accuracy. The SLA printer produced a smoother surface, but resulted in the distortion of thin features $(<1 \mathrm{~mm})$. MJ printers, on the other hand, produced comparable surface roughness and dimensional accuracy. However, ProJet MJP 3600 produced sharper edges when compared to the Objet 260 that produced round edges.

Originality/value - This paper, for the first time, provides a comprehensive comparison of three different commonly used 3DP technologies in terms of their dimensional capability and surface roughness without farther post-processing. Thus, it offers a reliable guideline for design consideration and printer selection based on the target application.
\end{abstract}

Keywords Measurement, 3DP, Dimensional capability, Polymer materials

Paper type Research paper

\section{Introduction}

$3 \mathrm{D}$ printing (3DP), also known as additive manufacturing (AM), rapid prototyping or solid freeform fabrication, was first introduced in the early 1980s by Charles Chuck Hall (Hull, 1986). In this work, AM and 3DP are interchangeably used. As its initial introduction, this technology has been rapidly developed, improved, diversified and widely applied in various fields as an alternative manufacturing approach. 3DP technologies have significantly evolved to include a broader range of applications spanning from aerospace and automotive to biomedical applications (Gu et al., 2015). The current 3DP technologies can be categorized based on the nature of the raw material used in the print process (Chua and Leong, 2017), which can be either solid, liquid or powder.

When compared against the conventional manufacturing processes (e.g. subtractive manufacturing [SM]), 3DP offers a broader range of freedom in terms of design and material flexibility (Hawaldar and Zhang, 2018). Moreover, the rise of

The current issue and full text archive of this journal is available on Emerald Insight at: www.emeraldinsight.com/1355-2546.htm

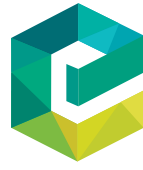

Rapid Prototyping Journal

25/5 (2019) 915-924

Emerald Publishing Limited [ISSN 1355-2546]

[DOI 10.1108/RPJ-03-2019-0061] composite printing technologies allows printing parts with thermo-physical properties that are similar to the ones obtained through the conventional manufacturing process (e.g. SM) (Agarwal et al., 2018). Consequently, 3DP applications have transitioned from a simple prototyping alternative to a complete end-product manufacturing solution (Campbell et al., 2011) offering a competitive advantage of on-demand manufacturing and/or make-toorder and/or on-site Manufacturing. Moreover, it offers a sustainable way of manufacturing for a wide variety of products by reducing or even eliminating extended and expensive supply-chains (Burkhart and Aurich, 2015; Ford and Despeisse, 2016; Jiang et al., 2017; Kellens et al., 2017; Klahn et al., 2015; Unkovskiy et al., 2017) in addition to its low cost and flexibility that have reinforced manufacturer's

\footnotetext{
(C) Younss Ait Mou and Muammer Koc. Published by Emerald Publishing Limited. This article is published under the Creative Commons Attribution (CC BY 4.0) licence. Anyone may reproduce, distribute, translate and create derivative works of this article (for both commercial and non-commercial purposes), subject to full attribution to the original publication and authors. The full terms of this licence may be seen at http://creativecommons.org/ licences/by/4.0/legalcode
}

Received 8 March 2019

Revised 8 March 2019

Accepted 18 April 2019 
interest toward the employment of this technology even in their routine manufacturing process.

In this study, three different 3DP technologies with one solidand two liquid-based build materials/systems are compared:

- fused deposition modeling (FDM);

- stereolithography (SLA); and

- material jetting (MJ), respectively.

FDM is known to be the most affordable solution, thus far, hence, its popularity. This technology is based on solid material melting and extrusion (S. Scott Crump [Stratasys], 1992). SLA technology is based on liquid photo-sensitive resin polymerization (Hull, 1986; Jean-Claude et al., 1986). The resin is initially disposed of in a containing tank, then selectively exposed to either ultraviolet (U.V.) laser or a U.V. projector. The latter is also known as digital light processing technology. MJ technology, as suggested by its name, is based on acrylic photopolymer jetting and polymerization (Ross et al., 1985). However, as opposed to SLA, MJ consists of material jetting and polymerization. The models are built on top of a motorized build plate that moves in the z-direction upon each layer completion. The vertical resolution of the printed models is tightly correlated with the build plate motion steps.

Although most of these technologies have been used in a variety of manufacturing and prototyping applications, little is known about the disparity that may exist between them in terms of their dimensional capabilities such as dimensional accuracy, repeatability and surface roughness. Consequently, often the technology selection for a given application is mainly based on budget availability and recommendations from experts or providers rather than reliable guidelines for the build specification requirements.

Several studies have oriented their interest toward the comparison of various aspects of 3DP technologies such as power consumption (Baumers et al., 2010), prosthesis dimensional accuracy (Unkovskiy et al., 2017), surface roughness (root mean square) (Bae et al., 2017) to site, but a few. Other studies have evaluated the effect of various printer settings on the dimensional accuracy, surface roughness and mechanical properties of printed models obtained from a single technology (Farzadi et al., 2014; Ramli et al., 2018). Other studies compared various 3DP technologies and materials characteristics under radiological conditions such as computer tomography scans (CT) (Bibb et al., 2011). These comparative studies reflect the rising interest toward a better understanding of AM systems discrepancies and limitations. Allowing, thus, a better 3DP technology selection based on given project requirements and restrictions.

It is to note that, to date, there is no such uniform standard to evaluate the dimensional accuracy of three-dimensional printed object. Therefore, various groups have adopted different strategies to achieve this task. For instance, three-dimensional systems has developed the CHRISMAS-TREE ${ }^{\mathrm{TM}}$ object (Dickens et al., 1995), Pang et al. (1995) have developed the H-4 structure ) and other groups have adopted custom, complex, objects to include curved, angled, extruded and cut-through features (Fahad and Hopkinson, 2012; Mahesh et al., 2006). Most of the developed benchmarks were designed to evaluate the performance of a single 3DP technology. In this present study, three 3DP technologies were compared; consequently, a custom benchmark that can accommodate all the tested technologies limitations had to be designed (Figure 1).

In conventional manufacturing technologies, it is known that dimensional accuracy and surface roughness are important properties to be continuously measured and improved for final products, particularly for applications involving small critical features (Elliott et al., 2017; Withell et al., 2011). Hence, there is even a more urgent need for dimensional capability comparison of the available 3DP technologies to guide future users about their limitations and process windows to further facilitate the applicability of these technologies and equipment. To this aim, in this current study, a comparison for the dimensional accuracy and surface roughness features among FDM, SLA and MJ printing technologies are investigated. In Section 2, the experimental materials, equipment and measurement methodology are explained. In Section 3, results and findings are presented comparatively followed by Section 4 where findings are discussed along with major conclusions and recommended future work.

\section{Methodology, materials and conditions}

In this work, three different 3DP technologies, namely, FDM, SLA, MJP (multiJet printing) are compared (Table I). The comparison targeted the dimensional accuracy and surface roughness of a printed benchmark containing a variety of common features (Figure 1).

\subsection{Model design and printing}

The benchmark model was designed using SolidWorks (Dassault Systems SolidWorks Corporation) with the dimensions indicated in Figure 1. Briefly, the base was $50 \mathrm{~mm}^{2}$ with $5 \mathrm{~mm}$ thickness. On the top surface of the base, straight and curved geometries were added. The straight features, also called protrusions (PT) here, are subdivided into three groups; large (PTlg), medium (PTmd), and small (PTsm); of 3, 1 and $0.4 \mathrm{~mm}$ width, respectively. The PTlg and PTmd spacing were of $2 \mathrm{~mm}$ where the PTsm spacing was $1 \mathrm{~mm}$. In addition to the $P T, C T$ straight feature of $1 \mathrm{~mm}$ width and spacing were also included. The

Figure 1 The benchmark model

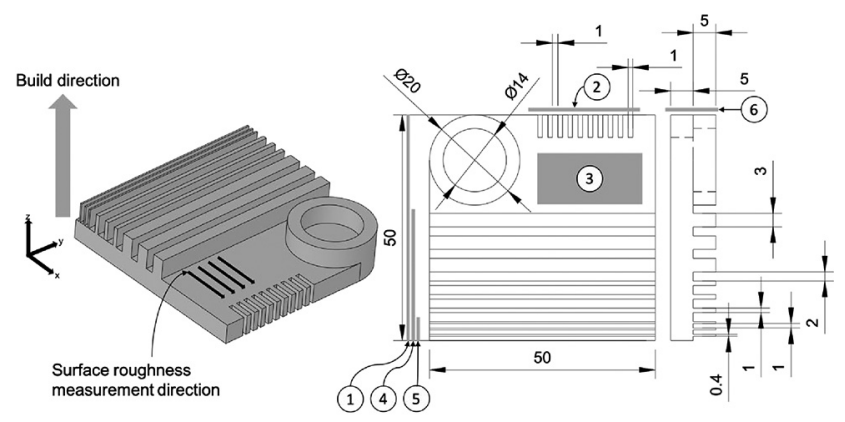

Notes: The 3D model was designed and oriented to prevent any support material generation (left panel), top view (middle panel), side view (right panel, 6). Top ROI (1), Cut Through (CT; 2), Roughness Region (RR; 3), Protrusions (PT; 4), small (sm) Protrusions PTsm (5). All dimensions are in millimeter ( $\mathrm{mm}$ ). The vertical arrow next to the benchmark shows the build direction, which was along the z-axis. The XYZ gizmo shows the orientation of the build plate 
Table I Tested equipment technical specification as described by each manufacturer's documentation

\begin{tabular}{|c|c|c|c|c|c|}
\hline Technology & Equipment & Material & Layer resolution $(\mu \mathrm{m})$ & $\mathrm{XY}$ resolution & Build volume $(\mathrm{W} \times \mathrm{D} \times \mathrm{H} \mathrm{mm})$ \\
\hline$\overline{\text { FDM }}$ & Ultimaker 3 extended & PLA & 200 & $12.5,12.5 \mu \mathrm{m}$ & $215 \times 215 \times 300$ \\
\hline SLA & Nobel $1.0 \mathrm{~A}$ & Resin & 25 & $130,130 \mu \mathrm{m}$ & $128 \times 128 \times 200$ \\
\hline \multirow[t]{2}{*}{ MJ } & Connex Objet260 & RGD840 & 12 & $600,600 \mathrm{dpi}$ & $255,252,200$ \\
\hline & ProJet MJP 3600 & VisiJet $囚$ M3-X & 32 & $375,450 \mathrm{dpi}$ & $298,185,203$ \\
\hline
\end{tabular}

curved features $(C F)$ included two concentric round protrusion with diameters of 14 and $20 \mathrm{~mm}$ (Figure 1). All features were with a $5 \mathrm{~mm}$ thickness. To prevent the supporting material generation, the benchmark did not include any overhanging regions. Thus, limiting any measurement biases that could result from the supporting material removal. The design was then exported as a binary STL file, which was sliced using each technology's slicing software as described below. A total of three parts were printed using each 3DP technology. All measurements on all geometric features were repeated three times per feature, thus totaling nine different measurements for the same type of feature, which offered a reasonable amount of statistical data to account for any variations.

\subsection{Fused deposition modeling model preparation and printing}

FDM printing was performed using the Ultimaker 3 Extended Printer (Ultimaker). Briefly, in FDM, plastic filaments are pushed (i.e. extruded) through a print head equipped with a heating element and a small diameter nozzle (up to $0.001 \mathrm{~mm}$ ). Once melted, the material is extruded through the printing nozzle. A thin string of filament is, then, obtained and deposited on the build plate layer-by-layer as instructed by the slicing data. Here, the printer was equipped with a $0.4 \mathrm{~mm}$ printing nozzle. The model was prepared using the manufacturer slicing software (Cura v3.0). Except for the height of the layers that was set to $0.4 \mathrm{~mm}$, all other printing parameters were kept at their default values. The models were printed using gray PLA. The printed model did not require any further post-processing steps and was used as is for the rest of the experimentation procedure.

\subsection{Stereolithography printing}

SLA printing was completed using the Nobel 1.0 A printer (XYZ Printing, Inc). In this SLA, a laser, emitting in the U.V. range, is used to induce polymerization and cross-linking of photopolymeric resins. A pair of galvanometers control the lateral motion of the laser beam. The tilt angle of each galvanometer is controlled by the slicing information contained in the "gcode" file, that is the file generated by the slicing software. Upon each layer completion, the build plate moves one layer-height and the process restart until the object is fully constructed. The benchmarks were sliced using the manufacturer's slicing Software (XYZware_Nobel v1.2.18). All slicing settings were kept at their default values (i.e. layer height $=0.1 \mathrm{~mm}$; quality= "good"; no brim; and no adhesion supports). We used the manufacturer blue resin as the build material.

\subsection{Material jetting printing printing}

The MJP prints were obtained using two printers, the Objet 260 Connex3 (Stratasys Ltd) and the ProJet MJP 3600 (3D Systems, Inc). Briefly, MJP printers work by depositing a microdroplet of polymers, which are flattened by a roller to produce a smooth layer. A U.V. light is used to induce polymerization. Upon polymerization, the build plate moves down one layer hight and the process starts over until full object completion. For the Objet 260, we have used the Objet Studio as a slicing software. Except for the print finish, which was set to glossy, all slicing settings were kept at their default values. We opted for VeroBlue (RGD840) as the build material. The required build plate adhesion layers were printed using SUP705 support material. The ProJet MJP 3600 models were prepared using the manufacturer's slicing software (threedimensional Sprint). Except for the printing resolution, which was set to high definition, all settings were kept at their default values. We used VisiJet ${ }^{\circledR}$ M3-X as a model material and the VisiJet ${ }^{\circledR} \mathrm{S} 300$ as support material.

\subsection{Model post-processing}

This current works aimed to compare the surface finish and dimensional accuracy of various 3DP technologies. Therefore, the model was designed and oriented to prevent support of material generation. However, some printing technologies require supporting material printing to simplify part removal from the build plate. For instance, the ProJet MJP 3600, inevitably, generated a small layer of support material underneath the printed object for ease removal. The printed object was incubated at $75^{3} \mathrm{C}$ for $30 \mathrm{~min}$ to melt away the support base. Similarly, the Object 260 printed generated an adhesion bed that was washed away using a high pressurized water jet. The model printed on the SLA machine was dipped in ethyl alcohol to remove excess resin and was, then, incubated in the U.V. oven for $30 \mathrm{~min}$ to ensure complete resin polymerization.

\subsection{Regions of interest on the model for measurements}

To the aim of our investigation, a total of six region of interest (ROI) were defined for measurements (Figure 1). The top ROI was used to measure the full object's width and height and the CF diameters [Figure 1(1)]. The side ROI was used to measure the object and extruded geometry thickness [Figure 1 (6)]. The edge ROI was used to measure the straight PT width and spacing [Figure 1(4)]. The PT geometries were subdivided into three groups as described in Table II. The corner ROI was used to measure the CT geometries width and spacing [Figure 1(2)].

\subsection{Measurements and analysis}

To accurately measure the dimensions of interest, the images of the models/parts were acquired using a dual pixel $12 \mathrm{MP}$ camera with $1.4 \mathrm{~mm}$ pixel size and a $1 / 20.55$ " sensor. The digital zoom was set to either $1 \mathrm{X}, 3 \mathrm{X}$ or $5 \mathrm{X}$ depending on the target geometry. For further details inspections, Zeiss Scope A1 microscope with a $5 \mathrm{X}$ objective was used. The raw images were acquired and saved using the Zen software (ZEISS, 
Table II Summary of PT features and sizes

\begin{tabular}{lcc}
\hline Geometries & Width $(\mathrm{mm})$ & Spacing $(\mathrm{mm})$ \\
\hline PTIg & 3.0 & 2.0 \\
PTmd & 1.0 & 2.0 \\
PTsm & 0.4 & 1.0 \\
\hline
\end{tabular}

Oberkochen and Germany). Dimensional analysis was performed using FIJI (a custom versions of ImageJ software, $\mathrm{NIH}$ ). Briefly, a $1 / 2 \mathrm{~mm}$ graduated ruler was used to setting the calibration unit. The ruler's image profile was plated and pickto-pick distances were evaluated for 10 graduations. The average was evaluated to 20.375 pixels $/ \mathrm{mm}$ and used for all the subsequent measurement. Each image was first converted into eight-bit images. Linear geometries were measured using a line tool that spans across the geometry's edges. The line tool allowed us to draw a line between tow edges and estimate the equivalent distance based on accuracy values estimated earlier. For better precision, the image profile was plotted to determine the geometry's edges better. Circular geometries were measured using the Feret diameters algorithm (Merkus, 2009) using a circle tool. This tool enabled us to draw a circle around a circular geometry and use it as the algorithm's input. As circles were used to evaluate the Feret's diameter, both the min- and the max-Fert were considered identical, and therefore, were included in the average calculation. For consistency, the width was measured along the x-axis, height along the y-axis and thickness along the z-axis. All dimensional values were represented as averaged deviations from nominals, which are all shown in Figure 1.

The surface roughness properties of the printed parts were measured using the SJ-201 surface test apparatus (Mitutoyo America Corporation). The measurements were performed on the top surface of the benchmark as shown in Figure 1. The apparatus was equipped with a standard detector (ref\# 178390) and the measurement traversing was set to $0.8 \mathrm{~mm}$. The surface roughness measurement included: Ra (arithmetic mean deviation), $\mathrm{Rz}$ (average distance between the highest peak and lowest valley) and $\mathrm{Rq}$ (root mean square). All measurements were repeated three times, and the resulted average, minimum and maximum values were calculated.

One-way ANOVA was applied for comparison between the measurement groups. When significant interactions were found, student's t-test was applied with $P<0.05$. Data are presented as Mean $\pm \mathrm{SD}$.

\section{Results}

\subsection{Surface roughness}

The surface roughness of the printed objects is expected to be different based on the applied printing technology because of

Figure 2 Surface roughness of the flat surface (ROI of 3 in Figure 1)

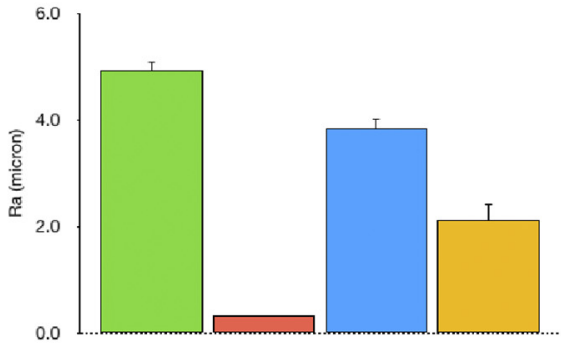

(a)

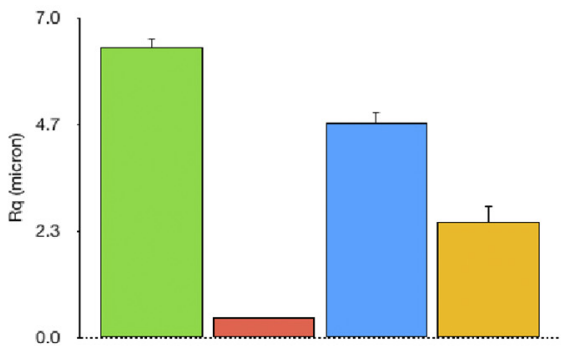

(c)

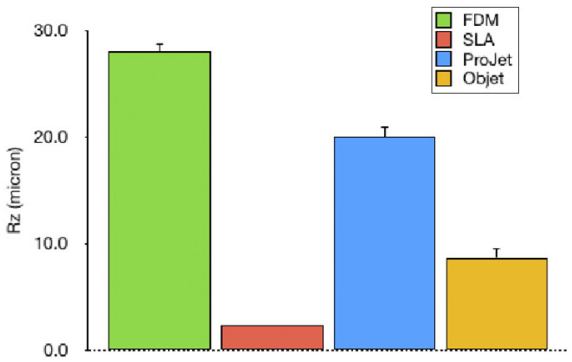

(b)

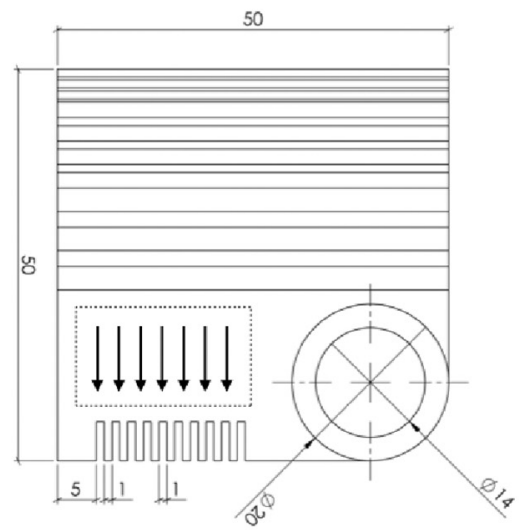

(d)

Notes: (a) Ra: arithmetic mean deviation; (b) Rz: average distance between the highest peak and lowest valley; (c) Rq: root mean square. The measurement region and direction are shown in (d). SLA results in the least surface roughness whereas FDM leads to rough surfaces 
the material used, its printing temperature and cooling rates and printing resolution. FDM showed a rough surface (Ra; $4.93 \mu m \pm 0.18)$ as expected, whereas the SLA technology produced significantly smooth surfaces $(\mathrm{Ra} ; 0.34 \mu m \pm 0.01)$. Both MJ printers showed intermediate values with Objet 260 producing smoother surfaces $(\mathrm{Ra} ; 2.12 \mu m \pm 0.31)$ and ProJet 3600 producing rougher surfaces $(\mathrm{Ra} ; 3.84 \mu m \pm 0.20)$. $\mathrm{Rq}$ and $\mathrm{Rz}$ parameters follow the same pattern as the $\mathrm{Ra}$ with a similar degree of significance (Figure 2).

\subsection{Dimensional measurements}

It is known that 3DP can facilitate the fabrication of highly detailed objects that can be composed of numerous small intricate geometries. These geometries may have a pure esthetic role such as emphasizing specific object's regions. However, in more advanced applications, these geometries might have a functional role. Consequently, their dimensional accuracy may be crucial in maintaining the proper function of the entire model. Thus, the dimensional accuracy of various geometries was evaluated.

To evaluate the overall dimensional accuracy of the printed parts, the part width and height were measured
[Figures 3(a)-3(b)]. For these dimensions, no significant difference between different 3DP technologies was observed, except for the parts printed using the ProJet 3600 that showed slightly, but negligible, smaller width and height. This observation might be the result of the heat treatment of the part that is required for support material removal. Accordingly, exposing the part to high temperatures $\left(70-80^{\circ} \mathrm{C}\right)$ then to room temperature $\left(\sim 25^{\circ} \mathrm{C}\right)$ could have induced shrinkage of the overall object. This phenomenon was also observed with the round geometries that showed smaller inner [Figure 3(c)] diameter and larger outer [Figure 3(d)] diameters.

\subsection{Protrusions geometries dimensions}

Measurements on the dimensions of the extruded features are presented in (Table I, Figure 4). Results do not indicate any significant difference between $3 \mathrm{DP}$ technology and equipment. Interestingly, with large geometries, a slight reduction in the spacing between each pin can be observed. However, this reduction does not reach significance due to the high error values $[-0.501 \mathrm{~mm} \pm-0.232$; Figure $4(\mathrm{~d})]$. Moreover, the

Figure 3 Overall benchmark dimensional measurement. Measurements were performed on images of benchmarks built with all tested printers (a; FDM, SLA, MJP, Objet260, from left to right, respectively)
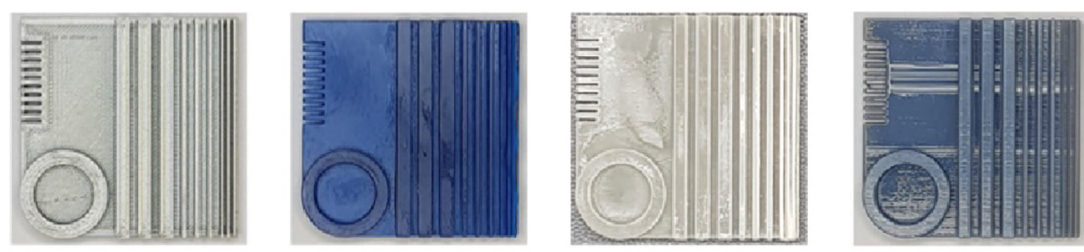

(a)

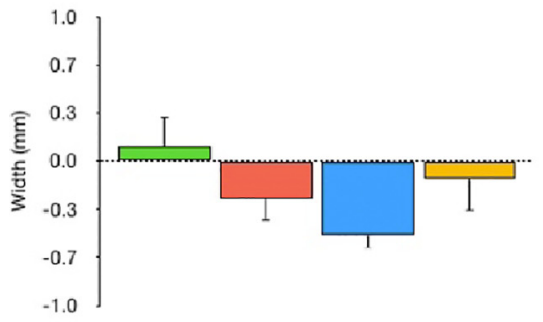

(b)

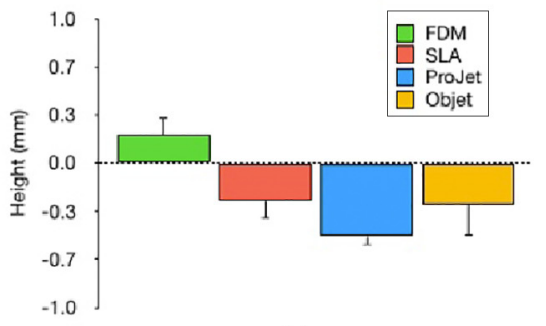

(c)

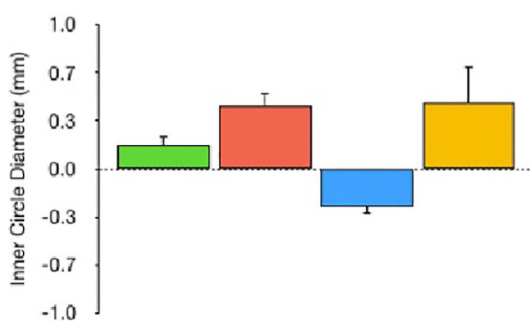

(d)

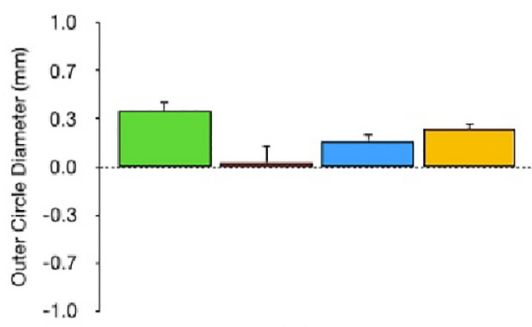

(e)

Notes: The width (a), height (b) and inner (c) and outer (d) circle diameters. The circle diameters were measured using a Feret diameter measurement, both the Feret min and max diameters were included in the average calculation. All data are represented as averaged deviations from nominal values were as follow: $50 \mathrm{~mm}$ for width and height, $14 \mathrm{~mm}$ for the inner diameter and $20 \mathrm{~mm}$ for the outer diameter 
Figure 4 PT geometries measurements

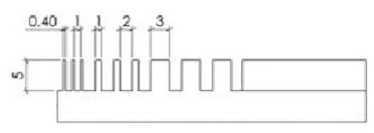

(a)

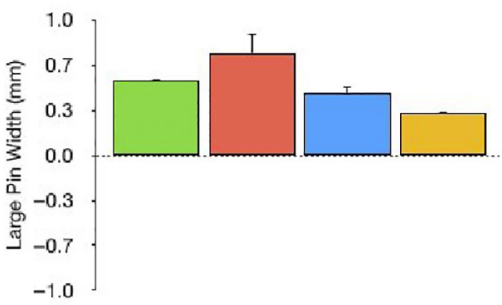

(c)

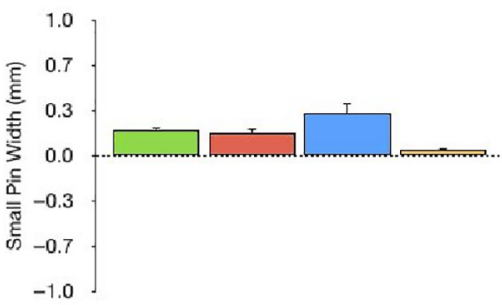

(e)

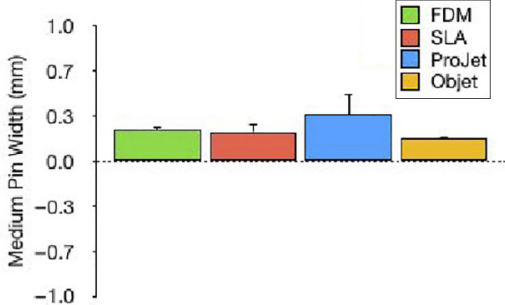

(b)

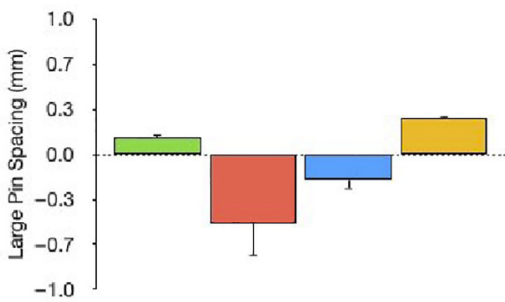

(d)

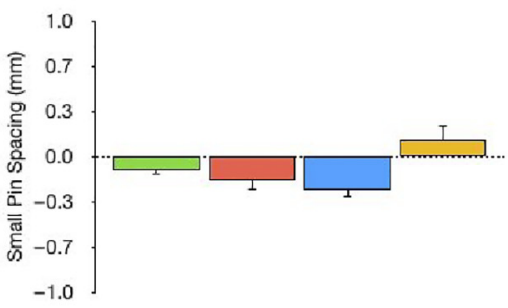

(f)

Notes: The drawing shows the location and design DIMENSIONS of the PT

(a). PT width and spacing of large (c) and (d), small (e) and (f). Both medium and large pin distances were identical. Hence, the absence of the medium pins spacing

reduced spacing can be correlated with the large width $[0.755 \mathrm{~mm} \pm 0.144$; Figure $4(\mathrm{c})]$. High measurement error (high STD) is mainly due to some residual resin that is difficult to clean correctly and is random from one part to the other.

\subsection{Thickness accuracy}

To evaluate the thickness accuracy, we included a $5 \mathrm{~mm}$ base upon which the geometries were built. All geometries were $5 \mathrm{~mm}$ thick. Our results did not show any significant difference between the thickness values of geometries in all printing technologies [Figure 5(b)]. In contrast, FDM and SLA technologies produced a significantly thicker base $(+0.545 \mathrm{~mm}$ \pm 0.061 and $+0.759 \mathrm{~mm} \pm 0.067$, respectively) [Figure 5(a)] This increased thickness could be the result of build plate adhesion layers. Indeed, both FDM and SLA technologies start the printing process by printing a few extra layers to improve object adhesion to the build plate. These layers are often difficult to remove.

\subsection{Measurements on cut-through geometries}

In addition to geometries constructed through positive extrusions, often 3DP objects require CT geometries that are constructed through negative extrusions. To evaluate the dimensional accuracy of such geometries, we have included 10 through all extrusion cuts to our model's edge with $1 \mathrm{~mm}$ width and spacing [Figure 6(d)]. ProJet MJP 3600 printers produced significantly wider pins $(+0.306 \mathrm{~mm} \pm 0.017)$ when compared against the designed model [Figure 6(a)]. This width oversizing was correlated with a lower pin spacing $(-0.255 \mathrm{~mm} \pm-0.025)$ [Figure 6(c)]. Similar to particular geometries described above, the ProJet dimensional discrepancy could be the result of residual support material and/or temperature cycles that the model is subject to during support material removal process.

\subsection{Thin features accuracy}

As mentioned earlier, the dimensional gaps observed between certain printed objects and the CAD model might be a result of geometric distortions of the printed objects due to severe heat cycles that the objects were exposed to during the post-processing step in which the temperature levels, durations and rates of these cycles are different. Indeed, post-processing steps including heat cycles and U.V. exposure might induce small geometries distortions. To verify the geometrical integrity of our model, we have measured the PT geometries at $5 \mathrm{X}$ digital magnification. 
Figure 5 Thickness measurement

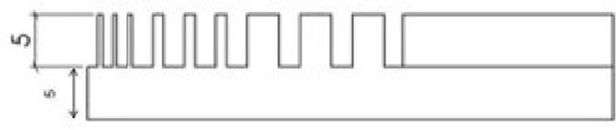

(a)

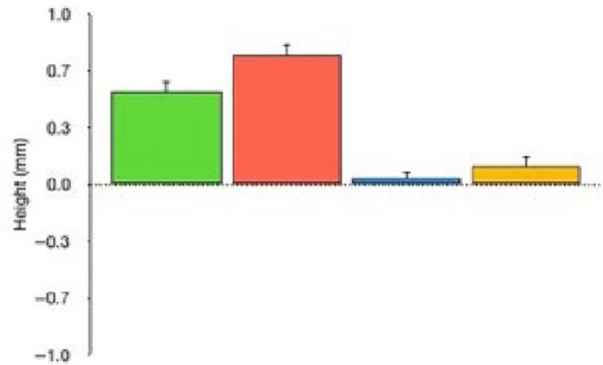

(b)

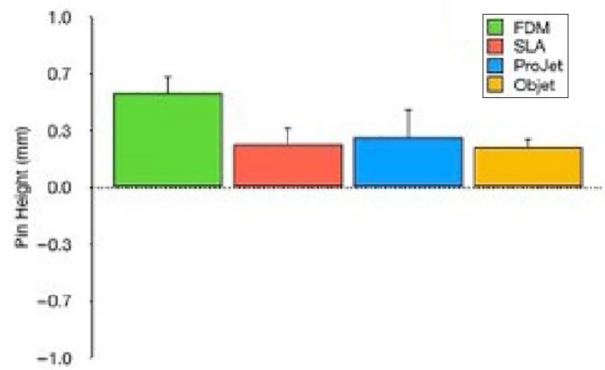

(c)

Notes: Comparison of deviations in the measured dimensions from the designed dimensions in the top panel (a): (b) total height of the sample, (c) height go the features, pins and channels

Figure 6 CT geometries measurement

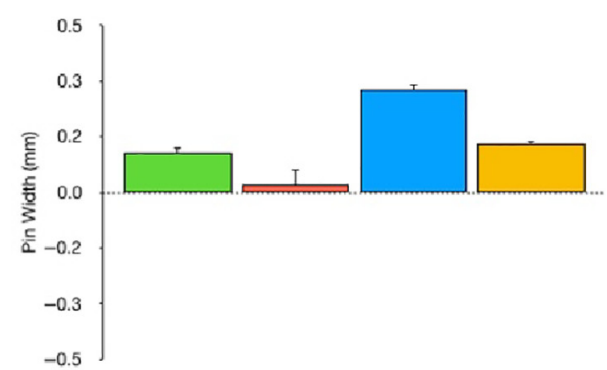

(a)

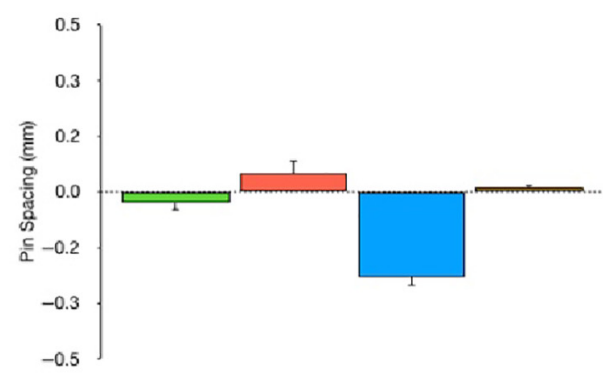

(c)

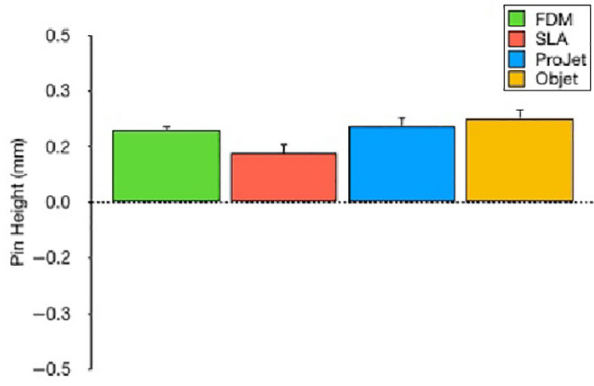

(b)

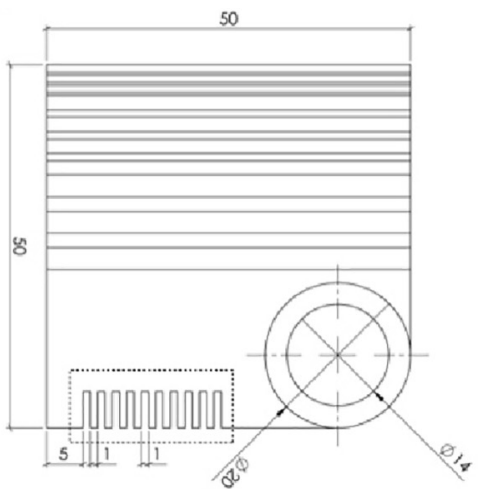

(d)

Notes: (a) ROI used to perform the measurement: (b) the measurements included: the pins spacing; (c) the pins width; (d) the pins height

Our data show that SLA object is subjected to significant geometric distortion, wobbling and waviness. That is particularly true for small geometries, which were designed with $0.4 \mathrm{~mm}$ width and $1 \mathrm{~mm}$ spacing (Figure 7 ).

\subsection{Edge sharpness and surface roughness}

In applications requiring fluid or air flow control, corner sharpness and high-quality surfaces need to be ensured mainly to control the flow regime, pressure and reduce residue 
Figure 7 Thin features geometric integrity

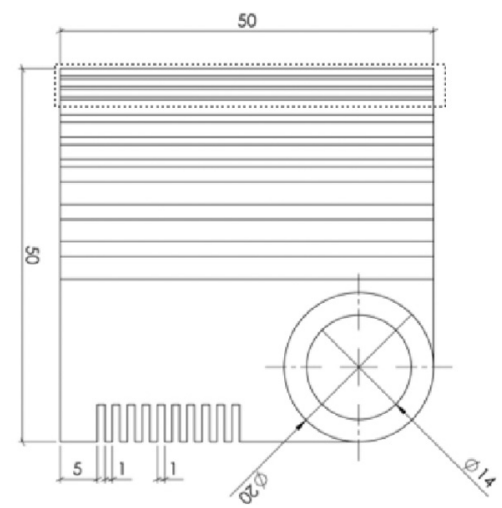

(a)

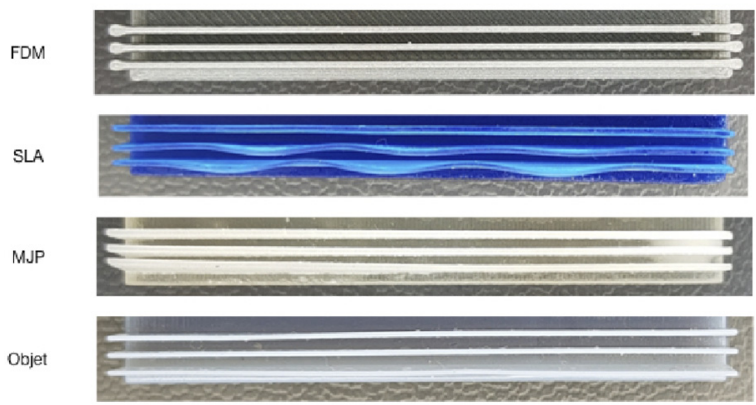

(b)

accumulation. The latter is particularly true for microfluidic applications that uses soluble particle and minerals. To investigate corner sharpness and surface roughness of our models, we have used a ZEISS microscope with $5 \mathrm{x}$ magnification objective to acquire CT geometries at the base, for corner sharpness observations and along the edge, for surface roughness observations (Figure 8). Our results showed that the Objet 260 printer $(\mathrm{MJ})$ to produce the smoothest surfaces. However, it produces curvy corners that were designed with $90^{\circ}$ angles (Figure 8; Objet) whereas, the ProJet MJP 3600 printers showed the opposite behavior. That is a rough surface with sharp corners (Figure 8; ProJet). The FDM printer, on the other hand, showed extreme curvy angles and surface roughness (Figure 8; FDM).

\section{Discussion and conclusions}

In this present work, three 3DP technologies (e.g. FDM, SLA and $\mathrm{MJ}$ ) and four equipment (Ultimaker 3 extended, Nobel 1.0 A, Connex Objet 260 and ProJet MJP 2600) were compared in terms of their dimensional accuracy, surface roughness and edge sharpness. To effectively compare the dimensional performance per se, we limited the post-processing steps to the minimum requirement. Our results showed significant variations between the compared technologies.

Although producing acceptable dimensional accuracy, FDM prints were characterized by a rough surface finish and a poor edge sharpness. It is to note, however, that our data were collected on the printed raw object. That is, without further finishing steps. As a conclusion, FDM prints have to be further
Figure 8 Microscopic assessment of edge sharpness and surface roughness of models obtained from FDM, SLA and MJ printing technologies. In the CAD design, the feature width and spacing were both set to $1 \mathrm{~mm}$

FDM
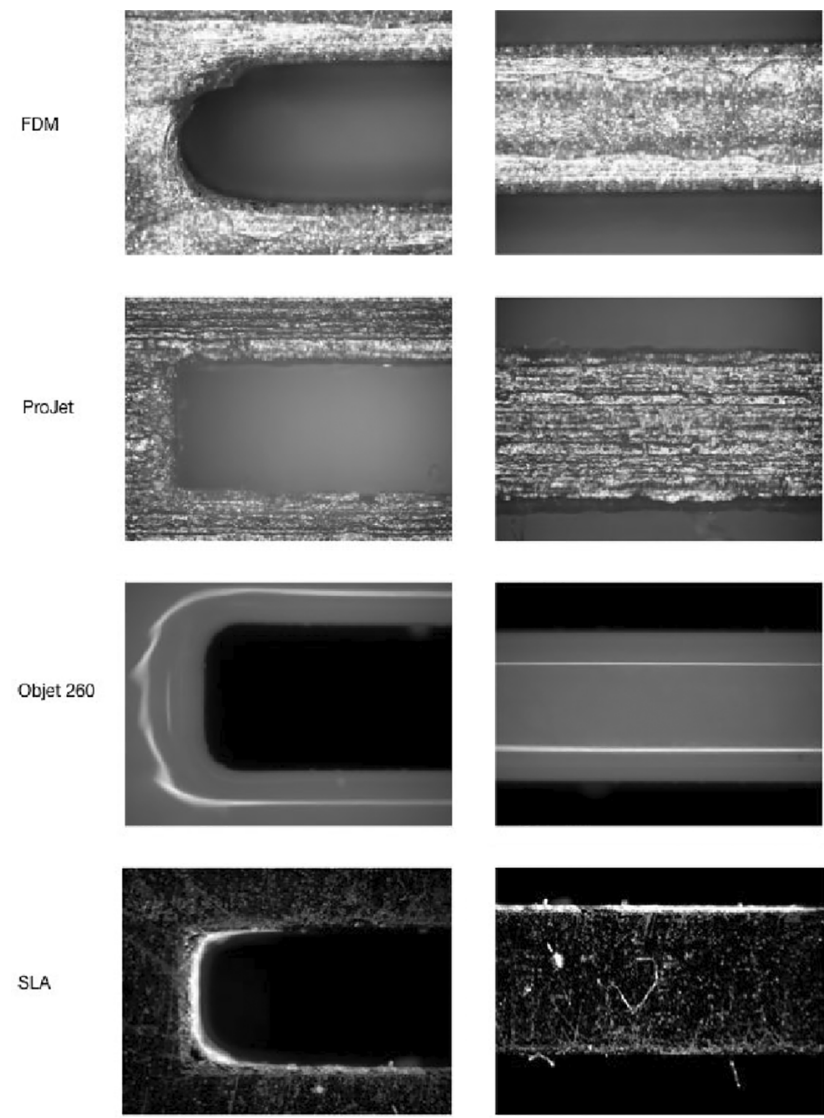

post-processed to improve their usability as end-product (Galantucci et al., 2009). When this is true for large features, it rapidly becomes cumbersome for small features. Thus, reducing the production rate and increasing waste production, which is against the sustainable aspect of AM. Various research groups are evaluating an alternative approach to limit the postprocessing phase. For instance, design considerations, slicing settings and object orientation could improve the resolution and surface roughness while reducing the post-processing steps (Agarwal et al., 2018; Khoshkhoo et al., 2018; Ramli et al., 2018). This is particularly important for parts where fit and form are essential and parts containing small, yet functional, geometries (Dickens et al., 1995). Despite these limitations, FDM technology is by far the most investigated technology. Its low cost and open-source aspect are indeed the reason behind this rise of attraction. These studies included, surface roughness measurement and modeling (Boschetto et al., 2013; Turner and Gold, 2015), dimensional accuracy (Ramli et al., 2018), surface roughness and quality (Agarwal et al., 2018; Armillotta, 2006). In contrast, given their high cost and complexity, only a few studies addressed MJ dimensional accuracy and surface roughness (Elliott et al., 2017; Khoshkhoo et al., 2018). Similarly, there has been a lack of extensive evaluation of such parameters for SLA (Campanelli et al., 2007). 
The low-cost of desktop SLA printers makes them the direct competitors of FDM (Szykiedans and Credo, 2016). They are often characterized by higher print resolution and lower surface roughness. In the present work, although SLA produces the smoothest surfaces, it also results in significant thin features warpage or waviness. Consequently, these printers could not be effectively used in models that contain such thin features. Besides, the bottom surface, which is in contact with the build plate, is often characterized by rough characteristics. That is particularly true for both FDM and SLA technologies (Armillotta, 2006). Indeed, to improve build plate adhesion of printed models, these technologies, often, print adhesion layers before printing the actual object. Consequently, as observed here, the overall thickness of the printed models is increased. As a conclusion, FDM and desktop SLA printers could be an excellent choice for prototyping and visualization applications, for end-product manufacturing; however, these technologies need further improvement.

Given their cost and high specifications, as advertised by their respective manufacturers, MJP printers were expected to produce ready to use end-product prints. Surprisingly, both the tested MJP printers showed significant limitations when printing thin features. As observed in Figure 6, Objet 260 fails in printing sharp corners of $1 \mathrm{~mm}$ cut-through features. The ProJet MJP 3600, on the other hand, produces rough surfaces as observed in Figures 2 and 6. Moreover, the thin features corner sharpness, are significantly affected by a tiny support layer deposition as seen in Figure 6 (ProJet).

Moreover, both these MJP technologies initiate the printing process by printing a couple of support layers. Although these layers aim to facilitate objects removal upon print completion, it adds an extra layer of complexity during the post-processing phase. Moreover, the residual support material negatively affects the overall dimensional accuracy of printed models. As observed in Figures 2 and 6 (Object 260), Objet 260 printer produced acceptable surface roughness with superior finish when the model is printed in the glossy mode. As shown by Udroiu et al. the build type (glossy and matte) can significantly affect the surface roughness properties of the printed models (Udroiu and Mihail, 2009). In this study, we choose the glossy type to prevent excessive post-processing on the printed model.

As a conclusion, FDM and desktop SLA printers showed significant limitations in terms of surface roughness and thin features accuracy, respectively. Accordingly, these technologies can be used as low-cost solutions for applications that do not require superior surface quality and tight dimensional accuracies such as visualization, demonstration and training applications. Surprisingly, at the microscopic level, MJP printers showed significant weakness in terms of corner sharpness and surface roughness. When combined, the present data confirm that fact that the tested AM technologies are not yet ready to produce ready to use end-products and advanced post-processing phase is required to achieve this task.

\section{References}

Agarwal, K., Kuchipudi, S.K., Girard, B. and Houser, M. (2018), "Mechanical properties of fiber reinforced polymer composites: a comparative study of conventional and additive manufacturing methods", Fournal of Composite Materials, London, Vol. 52 No. 23, pp. 3173-3181.
Armillotta, A. (2006), "Assessment of surface quality on textured FDM prototypes", Rapid Prototyping Fournal, Vol. 12 No. 1, pp. 35-41.

Bae, E.J., Jeong, I.D., Kim, W.C. and Kim, J.H. (2017), “A comparative study of additive and subtractive manufacturing for dental restorations", Fournal of Prosthetic Dentistry, Vol. 118 No. 2, pp. 187-193.

Baumers, M., Tuck, C., Hague, R., Ashcroft, I. and Wildman, R. (2010), "A comparative study of metallic additive manufacturing power consumption", Solid Freeform Fabrication Symposium, Editorial Council for the Journal of Prosthetic Dentistry, 2009, pp. 278-288.

Bibb, R., Thompson, D. and Winder, J. (2011), “Computed tomography characterisation of additive manufacturing materials", Medical Engineering and Physics, Institute of Physics and Engineering in Medicine, Vol. 33 No. 5, pp. 590-596.

Boschetto, A., Giordano, V. and Veniali, F. (2013), "Surface roughness prediction in fused deposition modelling by neural networks", The International fournal of Advanced Manufacturing Technology, Vol. 67 Nos 9/12, pp. 2727-2742.

Burkhart, M. and Aurich, J.C. (2015), "Framework to predict the environmental impact of additive manufacturing in the life cycle of a commercial vehicle", Procedia CIRP, Elsevier B. V, Vol. 29, pp. 408-413.

Campanelli, S.L., Cardano, G., Giannoccaro, R., Ludovico, A. D. and Bohez, E.L.J. (2007), "Statistical analysis of the stereolithographic process to improve the accuracy", Computer-Aided Design, Vol. 39 No. 1, pp. 80-86.

Campbell, T., Williams, C., Ivanova, O. and Garrett, B. (2011), "Could 3D printing change the world? Technologies, potential and implications of additive manufacturing", Atlantic Council, available at: www.cbpp. uaa.alaska.edu/afef/Additive/MFG.pdf (accessed 18 March 2018).

Chua, C.K. and Leong, K.F. (2017), 3D Printing and Additive Manufacturing, WORLD SCIENTIFIC, available at: https:// doi.org/10.1142/10200

Dickens, P.M., Stangroom, R., Greul, M., Holmer, B., Hon, K.K.B., Hovtun, R. Neumann, R., Noeken, S. and Wimpenny, D. (1995), "Conversion of RP models to investment castings", Rapid Prototyping fournal, Vol. 1 No. 4, pp. 4-11.

Elliott, O., Gray, S., McClay, M., Nassief, B., Nunnelley, A., Vogt, E., Ekong, J., Kardel, K., Khoshkhoo, A., Proanao, G., Blersch, D.M. and Carrano, A.L. (2017), "Design and manufacturing of high surface area 3D-Printed media for moving bed bioreactors for wastewater treatment", Fournal of Contemporary Water Research E̋ Education, Wiley/Blackwell (10.1111), Vol. 160 No. 1, pp. 144-156.

Fahad, M. and Hopkinson, N. (2012), “A new benchmarking part for evaluating the accuracy and repeatability of additive manufacturing (AM) processes", International Conference on Mechanical, Production and Automobile Engineering, pp. 234-238.

Farzadi, A., Solati-hashiin, M., Asadi-eydivand, M., Azuan, N. and Osman, A. (2014), "Effect of layer thickness and printing orientation on mechanical properties and dimensional accuracy of 3D printed porous samples for bone tissue engineering”, Vol. 9 No. 9, pp. 1-14. 
Ford, S. and Despeisse, M. (2016), “Additive manufacturing and sustainability: an exploratory study of the advantages and challenges", fournal of Cleaner Production, Vol. 137, pp. 1573-1587.

Galantucci, L.M., Lavecchia, F. and Percoco, G. (2009), "Experimental study aiming to enhance the surface finish of fused deposition modeled parts", CIRP Annals, Elsevier, Vol. 58 No. 1, pp. 189-192.

Gu, Q., Hao, J., Lu, Y., Wang, L., Wallace, G.G. and Zhou, Q. (2015), "Three-dimensional bio-printing", Science China Life Sciences, Science China Press, Vol. 58 No. 5, pp. 411-419.

Hawaldar, N. and Zhang, J. (2018), "A comparative study of fabrication of sand casting mold using additive manufacturing and conventional process", The International Fournal of Advanced Manufacturing Technology, Springer, London, Vol. 97 Nos 1/4, pp. 1037-1045.

Hull, C.W. (1986), "Apparatus for production of threedimensional objects by stereolithography”, US Patent 4,575,330, 8 August.

Jean-Claude, A., Le Mehaute, A. and De Witte, O. (1986), "Dispositif pour realiser un modele de piece industrielle", available at: https://doi.org/FR2567668

Jiang, R., Kleer, R. and Piller, F.T. (2017), "Predicting the future of additive manufacturing: a delphi study on economic and societal implications of 3D printing for 2030", Technological Forecasting and Social Change, Vol. 117, pp. 84-97.

Kellens, K., Mertens, R., Paraskevas, D., Dewulf, W. and Duflou, J.R. (2017), "Environmental impact of additive manufacturing processes: does AM contribute to a more sustainable way of part manufacturing?", Procedia CIRP, Vol. 61, pp. 582-587.

Khoshkhoo, A., Carrano, A.L. and Blersch, D.M. (2018), "Effect of surface slope and build orientation on surface finish and dimensional accuracy in material jetting processes", Procedia Manufacturing, Vol. 26, pp. 720-730.

Klahn, C., Leutenecker, B. and Meboldt, M. (2015), "Design strategies for the process of additive manufacturing", Procedia CIRP, Vol. 36, pp. 230-235.

Mahesh, M., Wong, Y.S., Fuh, J.Y.H. and Loh, H.T. (2006), "A six-sigma approach for benchmarking of RP\&M processes", International fournal of Advanced Manufacturing Technology, Vol. 31 Nos 3/4, pp. 374-387.

Merkus, H.G. (2009), "Particle size measurements: fundamentals, practice, quality", PLOS ONE, Springer, available at: https://books.google.com/books?id=1Lx4GzA7AUC\&pg=PA15 (accessed 22 November 2018).

Pang, T.H., Guertin, M.D. and Nguyen, H.D. (1995), "Accuracy of stereolithography parts: mechanism and modes of distortion for a 'Letter-H' diagnostic part", Solid Freeform Fabrication Proceedings, pp. 170-180.

Ramli, F.R., Faudzie, M.S.M., Nazan, M.A., Alkahari, M.R., Sudin, M.N., Mat, S. and Khalil, S.N. (2018), "Dimensional accuracy and surface roughness of part features manufactured by open source 3D printer", fournal of Engineering and Applied Sciences, Vol. 13 No. 3, pp. 1139-1144.

Ross, R.A., John, D.M. and William, R.K. (1985), "Thermodynamics and hydrodynamics of thermal ink jets", Hewlett Packard fournal, Vol. 36 No. 5, p. 21.

S. Scott Crump (Stratasys) (1992), "Apparatus and method for creating three-dimensional objects”, 30 October.

Szykiedans, K. and Credo, W. (2016), "Mechanical properties of FDM and SLA low-cost 3-D prints”, Procedia Engineering, Vol. 136, pp. 257-262.

Turner, B.N. and Gold, S.A. (2015), "A review of melt extrusion additive manufacturing processes: II: materials, dimensional accuracy and surface roughness", Rapid Prototyping fournal, Vol. 21 No. 3, pp. 250-261.

Udroiu, R. and Mihail, L.A. (2009), "Experimental determination of surface roughness of parts obtained by rapid prototyping", Proceedings of the 8th WSEAS International Conference on Circuits, Systems, Electronics, Control \& Signal Processing, pp. 283-286.

Unkovskiy, A., Spintzyk, S., Axmann, D., Engel, E.M., Weber, H. and Huettig, F. (2017), "Additive manufacturing: a comparative analysis of dimensional accuracy and skin texture reproduction of auricular prostheses replicas", Fournal of Prosthodontics, Vol. 28 No. 2, pp. 1-9.

Withell, A., Diegel, O., Grupp, I. (2011), "Porous ceramic filters through 3D printing", in Virtual and ..., available at: http://books.google.com/books?hl=en\&lr=\&id=Q002_TiMJ $6 \mathrm{kC} \& \mathrm{oi}=\mathrm{fnd} \& \mathrm{pg}=\mathrm{PA} 313 \& \mathrm{dq}=$ Porous + Ceramic + Filters + through $+3 \mathrm{~d}+$ Printing \&ots $=$ kgnt $7 \mathrm{MIm} 4 \mathrm{z} \& \operatorname{sig}=4 \mathrm{~J} 1 \mathrm{NzHU}$ K8Ge6eJ7Hg8mcrzKslU4\%5Cnhttp://books.google.com/ books?hl=en\&lr=\&id=Q002_TiMJ6kC\&oi=fnd\&pg=PA3 $13 \& \mathrm{dq}=$ Porous + ceram

\section{Corresponding author}

Muammer Koc can be contacted at: mkoc@hbku.edu.qa 